\title{
A Novel Design Method of the Double Spectrum Night Vision System
}

\author{
QinYi \\ Xi'an University of Science and Technology School of Computer Science and Technology \\ Xi'an, Shannxi, China \\ saddyqin1120@163.com
}

Keywords: night vision, infrared night vision technology, image fusion,competitive, image fusion algorithm

Abstract: With the rapid development of the automotive market and growing sense of security, the requirement of people to automotive safety higher and higher. If used auto infrared night vision system, it can improve the view definition and reduce the occurrence of traffic accidents at night. The infrared night vision technology is the kind of photoelectric technology used photoelectric imaging device implements the night observation. Infrared imaging sensor identifies the target by detecting thermal radiation of the difference between target and background, so, it is not sensitive to the brightness changes of the sense and it's imaging resolution is low. It is not conductive to the human eye observation. For car safety driving, not only to find the road object and to determine the accurate position of this road object, so the high resolution image is needed. Optical imaging sensor resolution is higher and can provide the road object's details. The fusion of the infrared and visible light image is conductive to comprehensive the indicative of infrared image and the clarity of the visible light image, and it is very important to the safety driving.

This paper implemented the design about a kind of double spectrum car night vision system and described the novel competitive algorithm about image fusion used the system.

\section{Introduction}

With the rapid development of the market and strengthening of security awareness.the demand about the automobile safety technology is increasing more and more. But the existing automobile lighting systems has several serious problems about the horizon near and the effect poor under the rain, snow, and so on. In the night, if the driver used the headlight can only see the 30 meters in front of the things. But if there is infrared night vision instrument, it will be improve the clarity of vision and reduce the occurrence of the traffic-accidents at night.

Infrared night vision technology is a kind of photoelectric technology based on photoelectric imaging. It is divided into active infrared night vision technology and passive infrared night vision technology. Active infrared night vision technology which observes object by active radiation and reflect the infrared light. Passive infrared night vision technology which observes object by itself emitting infrared radiation. Passive infrared night vision finds the object according to temperature or thermal radiation difference between the object and the background or different part of the object. So, passive infrared night vision system has much advantages just as working under fog, rain, snow and far distance. And it can identify camouflage and anti-interference also become the development of night vision equipment ${ }^{[1]}$.

Infrared imaging sensor relies on detecting thermal radiation of the differences between the target and the background to identify the target. It is not sensitive to the brightness of the scene changes 
and its imaging clear degree is low. So it is not conductive to the human eye observation. For the car security, it is not only to discover and discriminant road objects but also determine the accurate position of them. So it will be ask for images with high resolution. Optical imaging sensor resolution is higher, can provide the road object details. Infrared and visible light image fusion is advantageous to the comprehensive indicative of infrared image and visible light image clarity. It is very important for car safety driving.

This paper describes and implements a design method about double spectrum car night vision system.

\section{A method about double spectrum car night vision system}

\section{System composition design}

The car night vision system working environment temperature is broad, especially in China, the temperature difference in the north and the south is very big. In the north, the car night vision system needs to work in temperature from 20 degree centigrade below zero to 30 degree centigrade, but in the south, the car night vision system needs to work in temperature above 60 degree centigrade. So,it requires the system can adapt to the temperature range of the whole.

Infrared thermal imaging is the use of infrared detector, optical imaging to adopt advances technology of the focal plane of the objective ${ }^{[2]}$ accept measured target infrared radiation energy and its distribution of reflected in the infrared in the infrared detector lens infrared radiant energy,amplified,converted to standard video signal through the display shows the infrared thermal image map.

The infrared thermal image map and heat distribution on the surface of the object is corresponding to the filed, but various parts of the measure target distribution of thermal infrared radiation signal is weak, lead to image effect compared with visible light imaging effect of lack of administrative levels feeling and stereo feeling. So in the process of the actual system design, in order to more clearly and effective for determining the characteristic information of the measured target the double spectral integration design method was adopted.

The whole system includes four parts: a double spectrum imaging module, a image receiving system, a image display terminal a control terminal. As figure 1 shows.Among them, the double spectrum imaging module is used to image visible light and infrared light and collect, process and fuse image data; the image receiving system is used to complete the receiving image; the image display terminal complete to the image display; the control terminal is used to control the imaging mode.

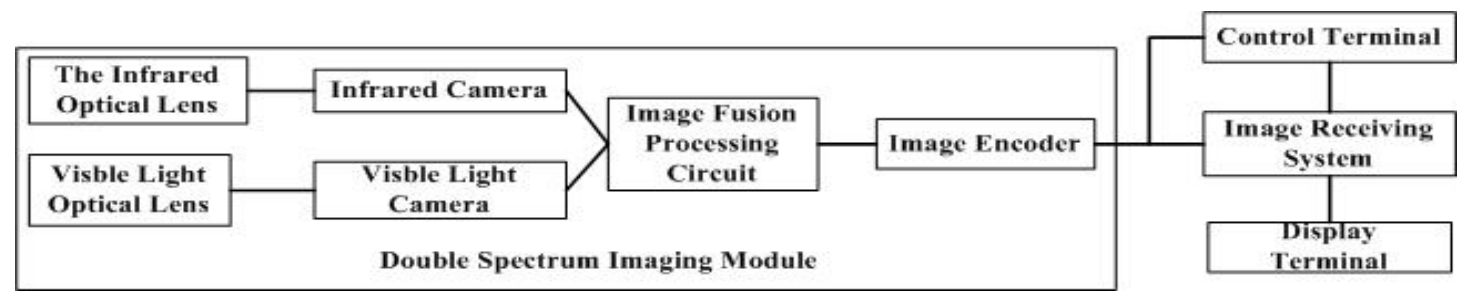

Figure 1 The Structure of Car Night Vision System

The system work process has several parts ${ }^{[3][4]}$. Firstly, the infrared optical lens, the infrared camera, the visible light optical lens and the visible light camera completed for infrared image and 
visible light image acquisition. Secondly, the visible and the infrared analog video signal which is registered and synchronized will be read in processing and fusion through the image fusion processing circuit. Thirdly, the fusion processing of data into the image encoder, through the video port receiving system output to the image, displayed on the display terminal. Among them, the core of the system is the double spectrum imaging module. Figure 2 shows the infrared imaging module structure, Figure 3 shows the image fusion processing circuit structure.

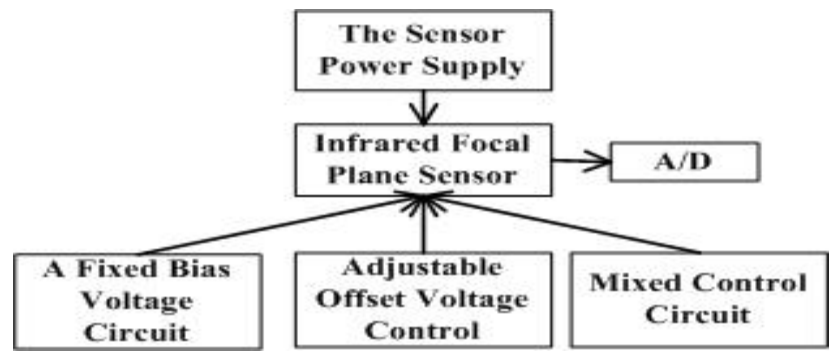

Figure 2 Infrared image module

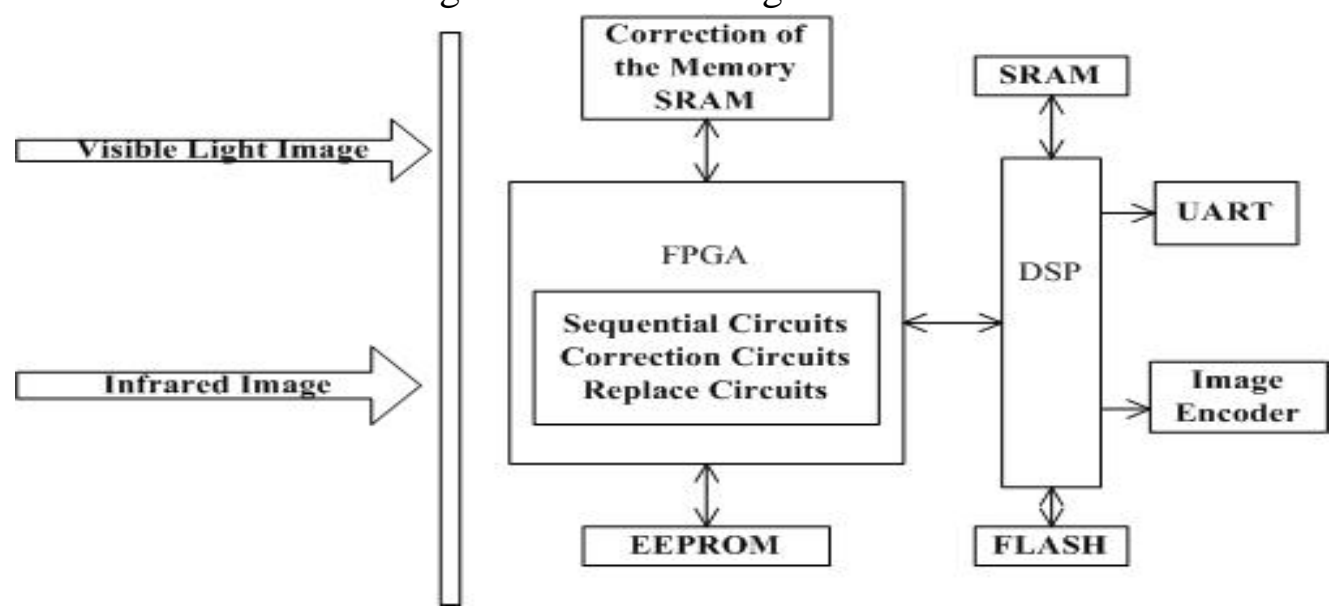

Figure 3 Image Fusion Processing Circuit

\section{System application}

The Double Spectrum car night vision system will be used the field without lighting, but if on the expressway, it shall be used all the process of driving either lighting or not. Especially in the following areas: the steep and long slope and foggy area.

Main components of this system and its application effect is shown in figuer 4, figuer 5 and figure 6:
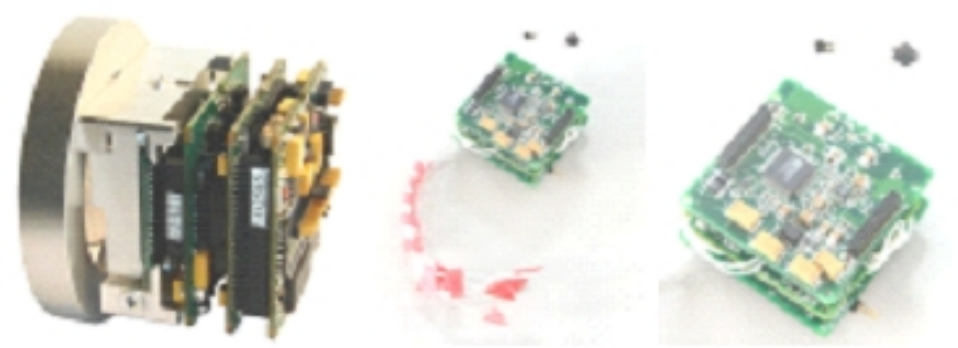

Figure 4 The night vision system movement 

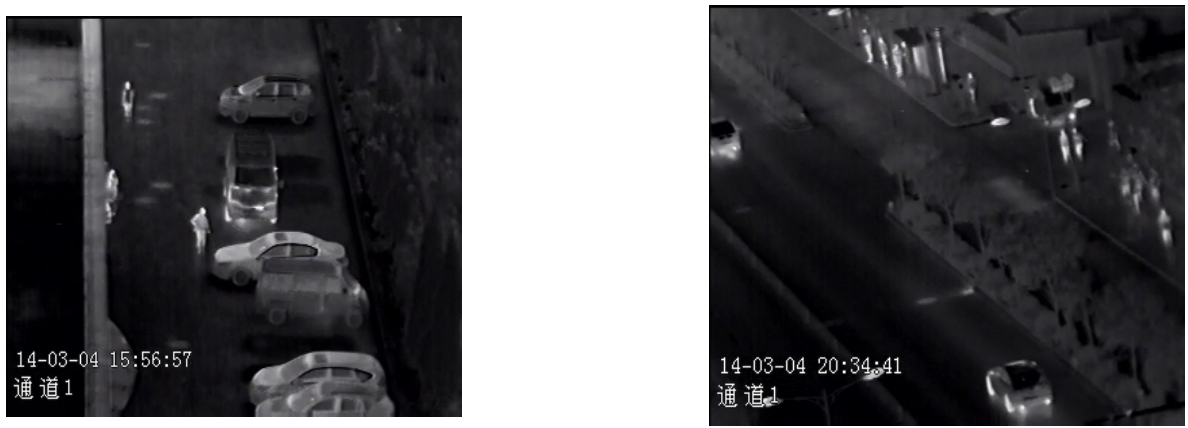

Figure 5 The night vision system imaging effect in sunny day

Figure 6 The night vision system imaging effect in night

\section{Usage of Image fusion algorithm competition}

Due to different imaging mechanism of multi-source images, the target position in different image has difference or distortion. In order to improve the fusion effect and enhance the target of image clarity, fusion algorithm research has called the focus of researchers ${ }^{[5]}$. At present, there are many mature image fusion algorithm, such as high-pass filtering method, the weighted average method, the wavelet transform method and based on evidence theory and neural network method and so on. But in the process of actual application, we will find these algorithm fusion performance is affected by the fusion of original image, it is that the fusion of different original image fusion algorithm using same, the resulting image quality will be different. So in the system described in this article the image fusion competition strategy is used.

The image fusion competition algorithm using the basic train of the thought is in the process of image fusion choose a variety of different fusion algorithms. These fusion algorithms using competition, during the process of fusion, the best algorithm at every stage will be selected dynamically.

Implementation steps are four parts ${ }^{[6][7][8]}$. Firstly, based on the system of visible light image and infrared image characters, choose a variety of different fusion algorithm fusion, the fusion of several different results are obtained. Secondly, the fusion result image quality analysis to determine the optimal fusion result and the corresponding optimal fusion algorithm. Thirdly, according to the optimal fusion result have not selected the other fusion algorithm was improved and repeat the above steps. Fourthly, untill obtain the optimal fusion results twice for the same fusion results and stop.

Through the image fusion algorithm competition, to achieve the optimal quality of the fused images and the corresponding fusion algorithm performance is stable.

The core idea of the image fusion algorithm competition is choice the best fusion image dynamically through some fusion algorithms working collaboratively. So it will get better results instead of simple to use specific formula. Compared with common fusion algorithm, this algorithm emphasizes to join the advantage information interaction into the fusion process and the advantage of this algorithm is the fusion image result is more stable and more quality.

\section{Conclusion}

The car night vision system has important value and significance in the field of technology level, safe driving level and market economic benefits.

Lower night-time driving safety hidden danger, to protect life and property safety 
According to statistics ${ }^{[9]}$, each year from traffic accident deaths reached 1 million, traffic accident deaths accounted for $70 \%$ of the accident at night. The night driving accident investigate its reason, in addition to shape and drive technology, the influence of the line of sight is not good enough at night is the main reason.

Has a broad market prospect

All kinds of advantages about car night-vision system are recognised by the driver.In the face of the domestic more than 85 million cars ${ }^{[10]}$, in the next five years, there will be at least $2-3 \%$ of the car is nearly 2.6 million will install the car night-vision system, this huge market prospect is broad.

In this paper, the design and method of double spectrum car night vision system is described. Including the part for the visible light and infrared imaging; the part for receiving, processing, and fusing of visible light and infrared image data; the part for image receive; the part for display terminal; the part for control imaging model and the part for switch control terminals. The system adopts the electronic fusion method can solve the problem of deviation of coaxial double spectrum image, can decrease the processing precision of imaging system. Also, the system can provide more clear image during driving. The system design is convenient for hardware implementation. The image fusion algorithm competition can enhance image details and reduce the computing complexity.

\section{Author's brief introduction and contact information}

Qin Yi, female (1978-), Dr, Xi'an University of Science and Technology School of Computer Science and Technology . Research direction: computer system structure, information fusion, and reliability. Tel:18092731892. Email:saddyqin1120@163.com

\section{Acknowledgments}

Scientific Research Program Funded by Shaanxi Provincial Education Department (Program No.14JK1469)

\section{References}

[1]Cai Zhan-en, Liu Chao-hui, Huang Jing. Optical design for low-level-light color night vision system and its image quality evaluation[J] .Journal of Applied Optics.,2010, Vol.31(5)

[2]Jin Ming-lei, Jin Wei-qi, Liu Chong-liang. Reference source for non-uniformity correction of the infrared focal plane arrays[J].Infrared Technology. 2012,Vol.34(7)

[3]ITT Night Vision: Enhanced night vision goggle(ENVG)AN/PSQ 20-ENVG (F6023)[EB/OL]. www.nightvision.com/products/military/ product_pages/ an_psq20-ENVG.htm.2009.

[4]Fluke Corp. Camera with visible light and infrared image blending[P].US, 7535002, 2009-5-19.

[5]Ma miao, Tian Hong-peng. Research on image cooperative fusion method [J]. Application Research of Computers. 2008,Vol.25(1)

[6]Maarten A. Hogervorst, et al. Method for applying daytime colors to nighttime imagery in realtime[C]//Proc of SPIE, 2008: 697403.

[7] Nikolaos Mitanoudis, Tania Stathaki. Optimal contrast correction for ICA-based fusion of multimodal images[J]. IEEE sensors journal, 2008,Vol.6(1): 1-21.

[8] Nikolaos Mitianoudis, Tania Stathaki. Optimal contrast for color image fusion using ICA 
bases[C]//IEEE International Conference on Digital Object Identifier, 2008: 1-7.

[9]http://www.tranbbs.com

[10]http://www.chinairn.com 